\title{
Editorial
}

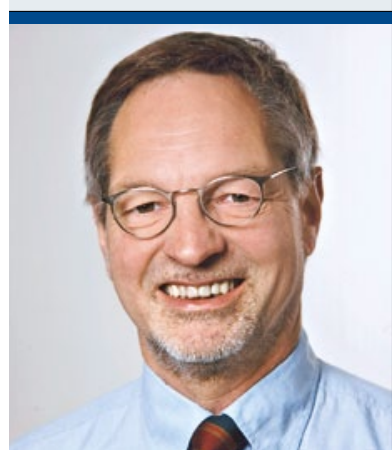

„Von meinen Patienten auf PET angesprochen, kann ich diese mit meinen hellseherischen Fähigkeiten verblüffen, wenn ich ihnen sage, was in ihrem Bericht mit Sicherheit stehen wird."

\section{Das dreckige Dutzend}

D ass das vergangene Jahr bei den Kassenpatienten auch für uns Urologen keinen spürbaren Honorarzuwachs gebracht hat, ist eine Binsenweisheit. Kein Wunder, dass nach einer Überlebensstrategie gesucht wird. Diese scheint zunehmend auf zwei Säulen zu stehen: dem zusätzlichen Angebot von Leistungen außerhalb der RVO-Erstattungen sowie dem Anstreben einer Umverteilung aus dem Honorartopf zu eigenen Gunsten. Letzteres schließt den Versuch ein, an einem Sonderhonorarpool zu partizipieren, denn das zur Verfügung stehende Geld bleibt begrenzt.

Die AOK selbst geht, was die letztgenannte Strategie betrifft, mit bestem Beispiel voran und schickt ihre Mannen in die Praxen, auf dass sie uns zeigen, wie eine richtige Kodierung zu laufen hat. Denn es ist schon ein Unterschied, ob bei der Diagnose Prostatakarzinom 1.900 Euro an die Kasse fließen oder ob bei in mindestens zwei Quartalen erfolgter Kodierung von Lymphknotenmetastasen die Kasse hierfür zusätzlich circa 8.000 Euro im Jahr erhält (Anmerkung: Niemand ist verpflichtet, diese AOK-Kodierungsspezialisten in seine Praxis zu lassen und seine Krankenakten für sie zu öffnen). Das Argument der AOK, dass die Mehreinnahmen schließlich auch uns zugute kommen würden, erscheint wenig glaubhaft, wenn man bedenkt, dass die Kassen in einem Spitzengespräch mit der KV Bayern in den jüngsten Verhandlungen Forderungen nach einem Hororarplus für die Vertragsärzte eine Absage erteilt haben und eine Nullrunde fordern, obwohl die bayerischen Ärzte beispielsweise bei der Verordnung von Arzneimitteln deutliche Einsparungen erzielt hatten (Quelle: Aktionsbündnis Urologie Bayern; ABU).

Für das Zusatzangebot von Leistungen außerhalb der RVO ist die Positronenemissionstomografie (PET) beim Prostatakarzinom ein gutes Beispiel, auch wenn ich davon ausgehe, dass hier kein „,cash-back“ von den Radiologen zu den Urologen stattfindet. Von meinen Patienten auf PET angesprochen, kann ich diese mit meinen zu $100 \%$ zutreffenden hellseherischen Fähigkeiten verblüffen, wenn ich ihnen sage, was in ihrem Bericht mit Sicherheit stehen wird: „Zum Ausschluss eines Prostatakarzinoms sollte (jedoch) sicherheitshalber eine Biopsie erfolgen." Und genau diese Biopsie wollte der Patient durch die PETDiagnostik vermeiden und trotzdem auf der sicheren Seite sein.

Dies führt uns zu einer Liste von Unsinnigkeiten beim Umgang mit dem Prostatakarzinom, die Prof. Lothar Weißbach, die besinnliche vorweihnachtliche Zeit nutzend, für die URO-NEWS-Leser unter seinem nicht veröffentlichten Arbeitstitel „Das dreckige Dutzend" erstellt hat (siehe Seite 24).

Die Punkte bieten eine gute Grundlage, zum Jahreswechsel Inventur zu machen und eventuell gute Vorsätze für das neue Jahr zu fassen. Das Beste daran: Jeder Punkt basiert auf geprüften Literaturstellen, die wir deshalb in Ausnahme zu unserer sonstigen Gepflogenheit („Literatur beim Verfasser“) im Einzelnen angeben. Vielleicht denkt dabei auch der eine oder andere Leser, dass zwölf Punkte gut zu den zwölf Monaten des neuen Jahres passen, in welchen man sich dann jeweils mit einer der angegebenen Literaturstellen befassen könnte.

An dieser Stelle wünscht Ihnen das URO-NEWSTeam ein gutes und erfolgreiches Jahr 2012!

Ihr

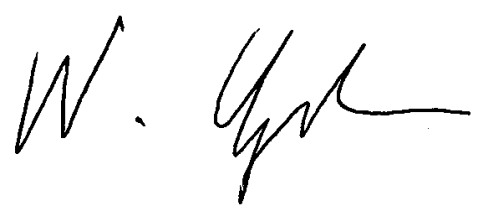

